\title{
Prior (Co)Variances Can Improve Multiple-Trait Across-Country Evaluations of Weakly Linked Bull Populations
}

\author{
T. Mark, ${ }^{1}$ P. Madsen, ${ }^{2}$ J. Jensen, ${ }^{2}$ and W. F. Fikse ${ }^{1}$ \\ ${ }^{1}$ Interbull Centre, Department of Animal Breeding and Genetics, Swedish University of Agricultural Sciences, \\ P.O. Box 7023, 75007 Uppsala, Sweden \\ ${ }^{2}$ Danish Institute of Agricultural Sciences, Research Centre Foulum, P.O. Box 50, 8830 Tjele, Denmark
}

\section{ABSTRACT}

National genetic evaluation results for fore udder attachment from 9 Ayrshire populations were used to assess the impact of different uses of prior genetic correlations in multiple-trait across-country evaluations (MACE) on predicted international genetic merit. These Ayrshire populations were poorly connected; that is, 2\% of the bulls had evaluations in 2 or more countries. Genetic correlations from the Holstein populations in the same countries were used as prior information to improve inferences of location parameters and international genetic merits. Fully Bayesian analyses using Gibbs sampling and computationally less demanding traditional MACE assuming a weighted average of prior and estimated Ayrshire genetic correlations were compared for 3 different prior degrees of belief and for different groups of bulls. Posterior means of genetic correlations estimated by Gibbs sampling were on average higher $(+0.2)$ than those estimated by REML. Posterior heritabilities differed up to 0.2 units from those assumed in national genetic evaluations. Predicted genetic merit and international sire rankings of bulls with daughter information in the country of interest were not affected substantially by method of analysis and even less by varying prior degree of belief. Method of analysis had a larger impact on predicted genetic merit for bulls without daughter information in the country of interest. Here the average correlation between predicted genetic merit in different analyses ranged from 0.62 to 0.99 . The predictive ability for young and randomly chosen bulls favored Bayesian MACE. The prior degree of belief did not have much impact on sire rankings and predictive ability, but intermediate prior degree of belief tended to perform best. All MACE analyses yielded nearly unbiased predictions. Traditional MACE assuming a simple weighted average of prior and estimated Ayrshire genetic correlations has been

Received February 4, 2005.

Accepted May 16, 2005.

Corresponding author: Thomas Mark; e-mail: Thomas.Mark@ hgen.slu.se. implemented by Interbull for routine international genetic evaluations.

(Key words: Bayesian multiple-trait across-country evaluations, prior, predictive ability, Ayrshire conformation)

Abbreviation key: AI-REML = average-information REML, BMACE = Bayesian MACE, CB = number of bulls with multiple evaluations, EDC = effective daughter contributions, EM-REML = expectation-maximization REML, MACE = multiple-trait across-country evaluations, $\mathbf{M S E}=$ mean square error, $\mathbf{P D B}=$ prior degree of belief, PEV = prediction error variance, TMACE $=$ traditional MACE.

\section{INTRODUCTION}

International genetic evaluations of dairy sires are currently conducted using multiple-trait across-country evaluations (MACE; Schaeffer, 1994), where similar traits measured in different countries are considered as different, but correlated traits. The necessary across-country genetic correlations are often difficult to estimate due to weak genetic ties between dairy populations in different countries, as the majority of bulls only have daughters in one country. As an example, weak connectedness is a severe problem for the international Ayrshire cattle population and especially so for Ayrshire conformation traits (Klei and Lawlor, 2001). This has delayed implementation of international genetic evaluations for these traits.

Inferences on international genetic merit might be improved by incorporating prior information about (co)variance components, for example, from other breeds. Bayesian methodology provides an elegant framework to incorporate prior information. Posterior distributions of dispersion parameters and predicted genetic merit can be sharpened by incorporating prior information that could otherwise be difficult to include. The prior degree of belief(PDB), however, depends on the specific problem and needs to be determined. Furthermore, Bayesian applications (e.g., via Gibbs sampling) are typically more computer demanding compared with BLUP methods that do not involve repeated sampling 
of random numbers. It is therefore interesting to investigate if approximate methods that are less computer demanding can achieve some of the expected gain in predictive performance from Bayesian methods.

Genetic evaluation methods including MACE have traditionally assumed genetic parameters to be known without uncertainty, although this is never the case in practice. The effect of ignoring uncertainty of variance components is small when the posterior distributions of the variance components are peaked (Gianola and Fernando, 1986; Schenkel et al., 2002). This is not necessarily the case when the data structure is poor. In such situations, the uncertainty of (co)variance components is typically large, and inference of genetic merit in a Bayesian setting can be useful, because it allows evaluating the impact of ignoring uncertainty of (co)variances on predicted genetic merit.

The aim of this study is to present a Bayesian MACE method and to study the impact of different uses of prior information in MACE for weakly connected populations on predicted international genetic merits. The predictive ability of different MACE approaches considering prior information with varying PDB will be compared for different groups of bulls.

\section{MATERIALS AND METHODS}

\section{Ayrshire Data}

National genetic evaluation results for fore udder attachment from 9 Ayrshire populations were used. These countries were Australia, Canada, Denmark, Finland, United Kingdom, Norway, New Zealand, Sweden, and United States. The date of national evaluation ranged from May 2000 (Australia) to May 2002 (Canada) for the 9 countries. Animals were traced in different time spans in different countries. The United States and Canada had bulls with national genetic merit born as early as 1953 , whereas the oldest bull with predicted national genetic merit in the three Nordic countries was born from 1978 (Denmark) to 1982 (Norway).

\section{Data Edits}

Bulls were required to have daughters in at least 10 herds and to be sampled through an official artificial insemination scheme. A further requirement only applying to estimation of genetic correlations for traditional MACE was that bulls should belong to a wellconnected subset. This subset included bulls having evaluations in more than one country (i.e., common bulls) as well as bulls that belong to $3 / 4$-sib groups that have members with evaluations in more than one country.

\section{Weighting Factors}

Each country, except Australia and the United Kingdom, provided effective daughter contributions (EDC) computed according to Fikse and Banos (2001). For Australia and the United Kingdom, the number of daughters instead was used as weighting factor (however, referred to as EDC hereafter).

\section{Connectedness}

The amount of genetic ties and total number of bulls with data included in the MACE analyses are shown in Table 1. Connectedness was generally weak and the number of common bulls was unevenly distributed across country combinations. The 9 populations could be seen as 2 weakly connected "subpopulations" (i.e., Nordic vs. non-Nordic countries), but some countrypairs within these 2 subpopulations also had few genetic links (e.g., Denmark-Norway). Sweden had common bulls with all countries and was an essential link provider.

Table 1. Number of common bulls (below diagonal), common $3 / 4$-sib families ${ }^{1}$ (above diagonal), and total number of bulls with data (on diagonal) included in Bayesian multiple-trait across-country evaluation (MACE) and in prediction of breeding values with traditional MACE. ${ }^{2}$

\begin{tabular}{lcrrrrrrrr}
\hline & AUS & CAN & DNK & \multicolumn{1}{c}{ FIN } & GBR & NOR & NZL & SWE & USA \\
\hline AUS & 148 & 8 & 0 & 1 & 2 & 0 & 3 & 1 & 6 \\
CAN & 8 & 478 & 2 & 6 & 21 & 0 & 17 & 5 & 70 \\
DNK & 0 & 2 & 864 & 4 & 0 & 1 & 2 & 17 & 0 \\
FIN & 1 & 6 & 4 & 1453 & 1 & 3 & 5 & 29 & 2 \\
GBR & 2 & 26 & 0 & 1 & 189 & 0 & 11 & 1 & 13 \\
NOR & 0 & 0 & 1 & 2 & 0 & 1732 & 0 & 10 & 0 \\
NZL & 3 & 15 & 2 & 5 & 12 & 0 & 288 & 6 & 11 \\
SWE & 1 & 5 & 14 & 23 & 1 & 7 & 6 & 586 & 1 \\
USA & 6 & 61 & 0 & 2 & 13 & 0 & 10 & 1 & 175 \\
\hline
\end{tabular}

${ }^{1}$ Common bulls are not included when counting $3 / 4$ sibs.

${ }^{2}$ Country codes: AUS $=$ Australia, CAN $=$ Canada, DNK $=$ Denmark, FIN $=$ Finland, GBR $=$ United Kingdom, NOR = Norway, NZL = New Zealand, SWE $=$ Sweden, USA = United States . 


\section{The MACE Model}

The MACE model (Schaeffer, 1994) used in all analyses was:

$$
\mathbf{y}_{\mathrm{i}}=\mu_{\mathrm{i}} \mathbf{1}+\mathbf{Z}_{\mathrm{i}} \mathbf{Q} \mathbf{g}_{\mathrm{i}}+\mathbf{Z}_{\mathrm{i}} \mathbf{s}_{\mathrm{i}}+\mathbf{e}_{\mathrm{i}}
$$

where $\mathbf{y}_{\mathrm{i}}=$ vector of deregressed national evaluations for country i; $\mu_{\mathrm{i}}=$ fixed effect of country mean; $\mathbf{g}_{\mathrm{i}}=$ vector of genetic group effects; $\mathbf{s}_{\mathrm{i}}=$ vector of random sire effects; $\mathbf{e}_{\mathrm{i}}=$ vector of random residuals; $\mathbf{Z}_{\mathrm{i}}=$ matrix assigning observations to sire effects; and $\mathbf{Q}=$ matrix assigning sires in $\mathbf{s}$ to group effects in $\mathbf{g}$.

International genetic merit on the ith country scale was generally defined as $\mu_{\mathrm{i}} \mathbf{1}+\mathbf{Q g}_{\mathrm{i}}+\mathbf{s}_{\mathrm{i}}$. The distributional properties of the vectors $\mathbf{g}_{i}, \mathbf{s}_{\mathrm{i}}$, and $\mathbf{e}_{\mathrm{i}}$ will be described separately for Bayesian and traditional MACE in the following sections.

Deregression. National evaluation results were deregressed within country using a model that corresponds exactly to [1], except that equations were only formed for one country at a time (Jairath et al., 1998). Assumptions of $\left[\mathbf{g}_{\mathrm{i}}, \mathbf{s}_{\mathrm{i}}, \mathbf{e}_{\mathrm{i}}\right]^{\prime}$ were the same as for traditional MACE (i.e., distributional properties given in formula [11]).

Pedigree and genetic group definitions. The same pedigrees and rules for assigning unknown ancestors to genetic groups were used in all MACE analyses. Paternal relationships were traced as far back as possible, whereas maternal grand-dams were always treated as unknown and included in the genetic group definition. The pedigree file contained records of 5790 bulls and 1086 known paternal ancestors. Genetic groups were formed based on selection path, birth year of the bull, and country of first registration. A relaxed grouping strategy was used; that is, only 12 groups consisting of 398 to 1042 phantom parents were formed for all analyses. The reason for this was to avoid problems with groups having very little information when groups were treated as fixed effects and to reduce the impact of different treatment of group effects.

\section{Bayesian MACE}

Jensen and Madsen (2002) described and implemented a fully Bayesian MACE (BMACE) method using Gibbs sampling. The same procedure was used here except for minor modifications.

Likelihood function. In BMACE, the conditional distribution of $\mathbf{y}$ given the location parameters $\mu_{\mathrm{i}}, \mathbf{g}_{\mathrm{i}}$ and $\mathbf{s}_{\mathrm{i}}$ was:

$$
\mathbf{y} \mid \mu, \mathbf{g}, \mathbf{s}, \mathbf{R}_{0} \sim \mathrm{N}\left[\mu_{\mathrm{i}} \mathbf{1}+\mathbf{Z}_{\mathrm{i}} \mathbf{Q} \mathbf{g}_{\mathrm{i}}+\mathbf{Z}_{\mathrm{i}} \mathbf{s}_{\mathrm{i}}, \mathbf{R}\right],
$$

where $\mathbf{R}_{0}$ is a matrix of residual (co)variances $\left(\sigma_{\text {eii }}\right)$, and the elements $\left(\boldsymbol{r}_{\mathrm{kii}}{ }^{\prime}\right)$ in $\mathbf{R}$ was equal to $\sigma_{\text {eii }} / \mathrm{min}$ $\left(\mathrm{EDC}_{\mathrm{ki}}, \mathrm{EDC}_{\mathrm{ki}}\right)$ for a bull $\mathrm{k}$ in countries $\mathrm{i}$ and $\mathrm{i}^{\prime}$.

Genetic group effects were assumed "fixed" and were therefore completely confounded with $\mu_{\mathrm{i}}$. The constraint $\mu_{\mathrm{i}}=0$ was imposed to overcome the resulting dependency, and as a result, [2] can be rewritten using $\mathbf{h}_{\mathrm{i}}=$ $\mu_{\mathrm{i}} \mathbf{1}+\mathbf{g}_{\mathrm{i}}$.

Prior distributions. Proper uninformative priors for $\mathbf{h}_{\mathrm{i}}$ were uniformly distributed and the prior distribution for international genetic merit was multivariate normal:

$$
\begin{aligned}
& \mathrm{p}\left(\mathbf{h}_{\mathrm{i}}\right) \propto \text { constant } \\
& \mathrm{p}\left(\mathbf{s} \mid \mathbf{G}_{0}, \mathbf{A}\right)=\mathrm{N}\left(\mathbf{0}, \mathbf{G}_{0} \otimes \mathbf{A}\right)
\end{aligned}
$$

where $\mathbf{A}$ is the additive genetic relationship matrix relating bulls with their sires and maternal grandsires and $\mathbf{G}_{0}$ is the additive sire (co)variance matrix.

The conjugate prior sire and residual (co)variance matrices were assumed to follow inverse-Wishart distributions:

$$
\begin{aligned}
& \mathrm{p}\left(\mathbf{G}_{0} \mid v_{\mathrm{a}}, \mathbf{V}_{\mathrm{a}}\right)=\mathrm{W}_{9}^{-1}\left(v_{\mathrm{a}} \mathbf{V}_{\mathrm{a}}, v_{\mathrm{a}}\right) \\
& \mathrm{p}\left(\mathbf{R}_{0} \mid v_{\mathrm{e}}, \mathbf{V}_{\mathrm{e}}\right)=\mathrm{W}_{9}^{-1}\left(v_{\mathrm{e}} \mathbf{V}_{\mathrm{e}}, v_{\mathrm{e}}\right)
\end{aligned}
$$

where $\mathbf{V}_{\mathrm{a}}$ and $\mathbf{V}_{\mathrm{e}}$ are known scale parameters for sire and residual (co)variance matrices, respectively; and $v_{\mathrm{a}}$ and $v_{\mathrm{e}}$ denote the degree of freedom for the inverseWishart distributions and can be interpreted as the PDB for (co)variances. Here we assumed the same PDB for both the sire and residual (co)variance matrix (i.e., $v=v_{\mathrm{a}}=v_{\mathrm{e}}$ ).

Posterior distributions. The fully conditional posterior distributions of the covariance matrices were also inverse-Wishart:

$$
\begin{aligned}
& \mathbf{G}_{0} \mid \mathbf{y}, \mathbf{h}, \mathbf{s}, \mathbf{e}, \mathbf{R}_{0}, \sim \mathrm{W}_{9}^{-1}\left(v \mathbf{V}_{\mathrm{a}}+\mathbf{S}_{\mathrm{a}}, v+\mathrm{q}\right) \\
& \mathbf{R}_{0} \mid \mathbf{y}, \mathbf{h}, \mathbf{s}, \mathbf{e}, \mathbf{G}_{0}, \sim \mathrm{W}_{9}^{-1}\left(v \mathbf{V}_{\mathrm{e}}+\mathbf{S}_{\mathrm{e}}, v+\mathrm{n}\right)
\end{aligned}
$$

where $\mathbf{S}_{\mathrm{a}}=\mathbf{s}^{\prime} \mathbf{A}^{-1} \mathbf{s}, \mathbf{S}_{\mathrm{e}}=\mathbf{e}^{\prime} \mathbf{R}^{-1} \mathbf{e}$, q is the total number of bulls in $\mathbf{s}$, and $\mathrm{n}$ is the total number of bulls with at least one observation in $\mathbf{y}$. For each bull (k), residuals were imputed for those countries where the bull did not have records; that is, the missing residuals were sampled from $\mathrm{N}\left(\mathbf{R}_{\mathrm{mo}} \mathbf{R}_{\mathrm{oo}}^{-1} \mathbf{e}_{0}, \mathbf{R}_{\mathrm{mm}}-\mathbf{R}_{\mathrm{mo}} \mathbf{R}_{\mathrm{oo}}^{-1} \mathbf{R}_{\mathrm{om}}\right)$, where the residuals and residual variances pertaining to each bull ( $\mathbf{e}_{\mathrm{k}}$ and $\mathbf{R}_{\mathrm{k}}$ ) are partitioned according to whether residuals are missing (m) or not (o) (Van Tassell and Van Vleck, 1996): $\mathbf{e}_{\mathrm{k}}=\left[\begin{array}{l}\mathbf{e}_{\mathrm{m}} \\ \mathbf{e}_{\mathrm{o}}\end{array}\right]$ and $\mathbf{R}_{\mathrm{k}}=\left[\begin{array}{l}\mathbf{R}_{\mathrm{mm}} \mathbf{R}_{\mathrm{mo}} \\ \mathbf{R}_{\mathrm{om}} \mathbf{R}_{\mathrm{oo}}\end{array}\right]$, where 
$\mathbf{e}_{\mathrm{m}}$ is the subvector of missing residuals and $\mathbf{e}_{0}$ is the subvector of residuals for observed traits. Our specific data contained no information about residual correlations, because each bull had only one record per country. Thus, only the priors were replicated in the posterior residual correlations.

The fully conditional distributions for $\mathbf{h}$ and $\mathbf{s}$ were multivariate normal:

$$
\begin{aligned}
& \mathbf{s} \mid \mathbf{y}, \mathbf{h}, \mathbf{e}, \mathbf{G}_{0}, \mathbf{R}_{0} \sim \mathrm{N}\left[\tilde{\mathbf{s}},\left(\mathbf{Z}^{\prime} \mathbf{R}^{-1} \mathbf{Z}+\mathbf{G}^{-1}\right)^{-1}\right] \\
& \mathbf{h} \mid \mathbf{y}, \mathbf{s}, \mathbf{e}, \mathbf{G}_{0}, \mathbf{R}_{0} \sim \mathrm{N}\left[\tilde{\mathbf{h}},\left(\mathbf{Q}^{\prime} \mathbf{Z}^{\prime} \mathbf{R}^{-1} \mathbf{Z} \mathbf{Q}\right)^{-1}\right]
\end{aligned}
$$

where $\mathbf{G}=\mathbf{I} \otimes \mathbf{G}_{0}, \mathbf{I}$ is an identity matrix and where $\tilde{\mathbf{h}}$ and $\tilde{\mathbf{s}}$ satisfies

$$
\begin{gathered}
\left(\mathbf{Q}^{\prime} \mathbf{Z}^{\prime} \mathbf{R}^{-1} \mathbf{Z} \mathbf{Q}\right) \tilde{\mathbf{h}}=\mathbf{Q}^{\prime} \mathbf{Z}^{\prime} \mathbf{R}^{-1} \mathbf{y}-\left(\mathbf{Q}^{\prime} \mathbf{Z}^{\prime} \mathbf{R}^{-1} \mathbf{Z}\right) \mathbf{s} \\
\left(\mathbf{Z}^{\prime} \mathbf{R}^{-1} \mathbf{Z}+\mathbf{G}^{-1}\right) \tilde{\mathbf{s}}=\mathbf{Z}^{\prime} \mathbf{R}^{-1} \mathbf{y}-\left(\mathbf{Z}^{\prime} \mathbf{R}^{-1} \mathbf{Z} \mathbf{Q}\right) \mathbf{h}
\end{gathered}
$$

Implementation of Gibbs sampler. The BMACE model was fitted using a Gibbs sampling algorithm based on a single long chain. The algorithm was implemented in the general DMU software package (Madsen and Jensen, 2003). The implementation closely followed the Gibbs sampler described by Jensen et al. (1994). A simple blocking strategy (García-Cortés and Sorensen, 1996) was applied to improve mixing. All location parameters belonging to a bull were sampled simultaneously, whereas all other parameters were sampled using single site updating.

Convergence of the Gibbs chain was assessed by the method of batching taking auto-correlations into account and by visual inspection of selected variables. In all BMACE analyses, 10,000 samples were discarded as burn-in and 500,000 additional rounds with an interleave of 10 rounds were used for inferences; that is, 50,000 samples were available for inferences. Posterior densities were estimated by the nonparametric approach of Scott (1992).

Mean values for prior distributions of (co)variance components. Estimated genetic correlations for Holstein fore udder attachment as measured in the same countries were used to compute prior means for genetic covariances when available. These Holstein estimates were taken from the Interbull February 2002 routine run (Interbull, 2002). Norway was the only country for which Holstein estimates were not available. The prior mean genetic correlation between Norway and another country was set to the lowest estimated Holstein genetic correlation for the other country. Prior means of genetic covariances were calculated from the prior means of genetic correlations and sire variances. Expectation-maximization (EM) REML estimates from the well-connected subset of the Ayrshire data were used as prior means for sire variances.

Prior means for residual variances were calculated from the prior mean sire variances and the heritabilities used in national genetic evaluations. The prior mean residual covariances were all equal to zero.

\section{Traditional MACE}

In traditional MACE (TMACE), the joint distribution of the random variables $\left[\mathbf{g}_{\mathrm{i}}, \mathbf{s}_{\mathrm{i}}, \mathbf{e}_{\mathrm{i}}\right]^{\prime}$ given $\mathbf{G}_{0}$ and $\mathbf{R}_{\mathrm{i}}$ was assumed to be multivariate normal:

$$
\left[\begin{array}{l}
\mathbf{g}_{\mathrm{i}} \\
\mathbf{s}_{\mathrm{i}} \\
\mathbf{e}_{\mathrm{i}}
\end{array}\right] \sim\left[\left(\begin{array}{l}
\mathbf{0} \\
\mathbf{0} \\
\mathbf{0}
\end{array}\right),\left(\begin{array}{lll}
\mathbf{G}_{0} \otimes \mathbf{I} & \mathbf{G}_{0} \otimes\left(\mathbf{A} \mathbf{Q}^{\prime}\right) & \mathbf{0} \\
& \mathbf{G}_{0} \otimes \mathbf{A} & \mathbf{0} \\
\text { Symmetric } & & \mathbf{R}_{\mathrm{i}}
\end{array}\right)\right]
$$

Thus, the genetic group effects were assumed random unlike for the BMACE model [2]. Model [1] with the assumptions [11] is equal to the one currently used by Interbull for international genetic evaluations of dairy sires. All parameters are assumed known without uncertainty in TMACE. The within-country heterogeneity of residual variances in $\mathbf{R}$ was the same as assumed for BMACE; that is, weighting factors were the same, but residual variances were fixed according to assumed heritabilities. Reliabilities for TMACE were approximated with the information source method (Harris and Johnson, 1998). Prediction error variances (PEV) were calculated from the approximate reliabilities (REL) as $(1-\mathrm{REL}) \sigma_{\text {sire }}^{2}$, where $\sigma_{\text {sire }}^{2}$ is the sire variance.

REML estimation. Sire variances were estimated within country using a single-trait EM-REML procedure (Sullivan, 1999). The ratio between sire and residual variance was fixed according to heritabilities used in national genetic evaluations and in computation of weighting factors.

Genetic correlations across all country combinations were simultaneously estimated with an EM-REML algorithm applied to a reduced set of mixed model equations (Klei and Weigel, 1998) corresponding to the model used for international genetic evaluations. The iterative process was stopped when the relative change in $\gamma_{\mathrm{ij}}=\mathrm{G}_{\mathrm{ij}} / \sqrt{\mathrm{R}_{\mathrm{ii}} \times \mathrm{R}_{\mathrm{jj}}}$ or the relative change of elements in the genetic correlation matrix in subsequent rounds of iteration was less than $10^{-7}$ for all elements.

Weighted average of genetic correlations. The genetic correlations used in TMACE were not the pure Ayrshire estimates. Instead, a weighted average of estimated genetic Ayrshire $\left(\mathrm{r}_{\mathrm{Gays}}\right)$ and Holstein correlations $\left(r_{G h o l}\right)$ were used. The Holstein correlations used for this 
Table 2. Summary of traditional multiple-trait across-country evaluation (TMACE) and Bayesian multipletrait across-country evaluation (BMACE) runs with respect to assumptions about sire variances $\left(\sigma_{\mathrm{s}}^{2}\right)$, residual variances $\left(\sigma_{\mathrm{e}}^{2}\right)$, genetic correlations $\left(\mathrm{r}_{\mathrm{G}}\right)$, and prior degree of belief (PDB), respectively.

\begin{tabular}{llllll}
\hline Run & MACE & $\sigma_{\mathrm{s}}^{2}$ & $\sigma_{\mathrm{e}}^{2}$ & $\mathrm{r}_{\mathrm{G}}$ & PDB $^{1}$ \\
\hline $1,2,3$ & TMACE & REML $^{2}$ & Fixed $^{3}$ & REML $^{2}$ avg $^{4}$ & $d=\{8,4,2\}$ \\
$4,5,6$ & TMACE & REML $^{2}$ & Fixed $^{3}$ & Gibbs $^{5}$ & $v=\{5,10,100\}$ \\
$7,8,9$ & TMACE & Gibbs $^{4}$ & Gibbs $^{5}$ & Gibbs $^{5}$ & $v=\{5,10,100\}$ \\
$10,11,12$ & BMACE & Gibbs $^{6}$ & Gibbs $^{6}$ & Gibbs $^{6}$ & $v=\{5,10,100\}$ \\
\hline
\end{tabular}

\footnotetext{
${ }^{1} d$ are defined in formula [14] and $v$ in formula [4] and [5], i.e. \{low, medium, high PDB\}.

${ }^{2}$ Sullivan (1999).

${ }^{3}$ Calculated according to heritability assumed in national genetic evaluation.

${ }^{4}$ Weighted average of Ayrshire and Holstein REML estimates as described above.

${ }^{5}$ Mean posterior estimates.

${ }^{6}$ Fully Bayesian inference.
}

were identical to the prior mean genetic correlations used in BMACE.

Prior genetic correlations $\mathrm{p}\left(\mathrm{r}_{\mathrm{G}}\right)$ were combined with estimated Ayrshire correlations using Bayes formula: $\mathrm{p}\left(\mathrm{r}_{\mathrm{G}} \mid \mathbf{y}\right) \propto \mathrm{p}\left(\mathrm{r}_{\mathrm{G}}\right) \times \mathrm{p}\left(\mathbf{y} \mid \mathrm{r}_{\mathrm{G}}\right)$. These densities were all assumed to be Gaussian; that is, $\mathrm{p}\left(\mathrm{r}_{\mathrm{G}} \mid \mathbf{y}\right) \sim \mathrm{N}\left(\mathrm{r}_{\mathrm{G}}, \sigma_{\mathrm{rG}}^{2}\right), \mathrm{p}\left(\mathrm{r}_{\mathrm{G}}\right)$ $\sim \mathrm{N}\left(\mathrm{r}_{\mathrm{Ghol}}, d^{2} \sigma_{\mathrm{rGhol}}^{2}\right)$, and $\mathrm{p}\left(\mathbf{y} \mid \mathrm{r}_{\mathrm{G}}\right) \sim \mathrm{N}\left(\mathrm{r}_{\mathrm{Gays}}, \sigma_{\mathrm{rGays}}^{2}\right)$, where $d$ was a constant that can be varied to reduce the weight put on the prior information relative to the information from the Ayrshire data. Given these assumptions, the posterior mean (weighted) genetic correlation $\left(\mathrm{r}_{\mathrm{G}}\right)$ is given by the following equation:

$$
\mathrm{r}_{\mathrm{G}}=\frac{\frac{1}{\sigma_{\mathrm{r}_{\mathrm{ays}}}^{2}} \mathrm{r}_{\mathrm{G}_{\text {ays }}}+\frac{1}{d^{2} \sigma_{\mathrm{r}_{\mathrm{G}_{\text {hol }}}}^{2}} \mathrm{r}_{\mathrm{G}_{\mathrm{hol}}}}{\frac{1}{\sigma_{\mathrm{r}_{\mathrm{ays}}}^{2}}+\frac{1}{d^{2} \sigma_{\mathrm{r}_{\mathrm{h}}}^{2}}}
$$

It was further assumed that the standard error of the genetic correlation coefficient $\left(\sigma_{\mathrm{rG}}\right)$ could be approximated as being proportional to the reciprocal of the square root of the number of common bulls (CB) between the 2 countries ( $i$ and $j$ ) of interest:

$$
\sigma_{\mathrm{r}_{\mathrm{G}}}=\mathrm{k} / \sqrt{\mathrm{CB}_{\mathrm{ij}}}
$$

where $k$ was a constant and CB was required to be at least 1 . The constant $k$ was assumed equal for all country combinations regardless of breed in this study. The correlation between estimates obtained with [13] and asymptotic standard errors obtained with the averageinformation REML (AI-REML) of Madsen et al. (2000) was 0.89 for country-pairs with at least one CB. The standard errors obtained from AI-REML were not realistic when $\mathrm{CB}$ was zero due to violations of asymptotic theory.
Combining formulas [13] and [12] yields [14]:

$$
\mathrm{r}_{\mathrm{G}}=\frac{\mathrm{CB}_{\text {ays }} \mathrm{r}_{\mathrm{G}_{\text {ays }}}+\frac{\mathrm{CB}_{\text {hol }}}{d^{2}} \mathrm{r}_{\mathrm{G}_{\mathrm{hol}}}}{\mathrm{CB}_{\text {ays }}+\frac{\mathrm{CB}_{\text {hol }}}{d^{2}}}
$$

The CB for Holstein was always higher than the corresponding numbers for Ayrshire, except for Norway and some country-pairs involving Finland. The genetic correlation matrix was bended (Jorjani et al., 2004) before used in TMACE to ensure positive definiteness. In this bending procedure, the allowed changes in genetic correlations were inverse-proportional to $\mathrm{CB}_{\text {ays }}+\mathrm{CB}_{\mathrm{hol}} / d^{2}$.

\section{Analyses}

Twelve MACE runs were conducted (Table 2). The first 9 runs were all TMACE with different genetic parameters, whereas the last 3 runs were BMACE with varying $\mathrm{PDB}$ for (co)variance matrices.

Firstly, 3 different values were used for $d$ in formula [14]; namely 8, 4, and 2, representing low, medium, and high PDB (runs 1, 2, and 3, respectively). Similarly, 3 Gibbs sampling chains with different PDB (i.e., 5, 10, and 100 degrees of freedom, respectively) were conducted (runs 10 to 12).

Differences between runs 1 to 3 and runs 10 to 12 could be due to either different parameters or considering uncertainty of parameters. To pinpoint the reason for these differences, posterior means of parameters from BMACE were used in TMACE. Runs 4 to 6 used posterior means of genetic correlations from BMACE and assumed the same variance components as in runs 1 to 3 . Finally, 3 runs using posterior genetic correlations, sire variances and heritabilities from BMACE were conducted (runs 7 to 9). 


\section{Comparisons}

Predicted international genetic merits were standardized within country to the sire transmitting ability scale and expressed in sire standard deviation $\left(\sigma_{\text {sire }}\right)$ units for the country in question. This was done to ease interpretation and enable comparison of results across country scales. The estimates of the sire variances from run 1 were used for standardization in all analyses.

Comparisons of results from different MACE runs were often done separately for foreign and domestic bulls. Foreign bulls were defined as bulls included in MACE with zero EDC in the country in question, whereas domestic bulls were defined as bulls with 1 or more EDC in the country in question. Domestic bulls were either import or other domestic bulls. Import bulls were identified as bulls first tested in another country. Thus, import bulls were typically proven elite bulls with evaluations in multiple countries.

Pearson product-moment and Spearman rank correlations between predicted international genetic merit from different MACE runs were calculated for the top 100 ranking bulls in run 1 . Bulls with data and a minimum reliability of $40 \%$ in run 1 were considered to calculate these correlations.

Predictive ability. To assess the predictive ability of the alternative MACE approaches, the 12 MACE runs (Table 2) were repeated with selected subsets of certain national breeding values set to missing. Three different reduced data sets were defined within each country for investigating the predictive ability with respect to each of these 3 particular categories of bulls, respectively:

1) The youngest 5 percentage of bulls with data

2) A randomly selected 5 percentage of bulls with data

3) The youngest 25 percentage of import bulls with at least 40 daughters in 20 herds.

The national genetic merit was set to missing before the deregression for bulls belonging to the particular subset. This was done to avoid deregressing information that will not subsequently be regressed and thereby ensuring that the predicted genetic merits for the selected group of bulls were comparable with national genetic merit. In the first 2 reduced data sets, a total of 300 national genetic merits were set to missing across all countries, whereas only 27 national genetic merits were set to missing in the third data set due to relatively few bulls identified as imported.

The genetic parameters used in TMACE applied to a reduced data set were equal to those used in the corresponding TMACE applied to the full data set. For BMACE, this was not the case, because inference of genetic parameters and international genetic merit is done simultaneously.
The predicted genetic merits ( $\mathrm{TA}^{\text {red}}$ ) of the bulls set to missing in MACE applied to the reduced data set were compared with their national genetic merits $\left(\mathrm{TA}^{\text {nat }}\right)$ and with their predicted genetic merits $\left(\mathrm{TA}^{\text {full }}\right)$ from the corresponding MACE applied to the full data set.

The predictive ability of the models was accessed by 3 different statistics: 1) mean square error (MSE) of the differences between $\mathrm{TA}^{\text {red }}$ and $\mathrm{TA}^{\text {nat; }}$ 2) $\mathrm{b}_{\text {full }}$ red, the regression coefficient from the regressions of $\mathrm{TA}^{\text {full }}$ on $\mathrm{TA}^{\text {red; }}$ and 3) $\mathrm{r}_{\text {full,red, }}$, the correlation between $\mathrm{TA}^{\text {full }}$ and $\mathrm{TA}^{\text {red. }}$. These 3 statistics were calculated across all bulls set to missing in the concerned reduced data set and across all 9 countries. The summation across countries is appropriate because the sire variance of all country scales were standardized to 1.0 . The 3 statistics were computed for the 3 sets of bulls set to missing and for the 12 different MACE runs described in Table 2. The latter 2 statistics were computed for both the country scale where the national genetic merit was set to missing (d) and for the 8 other country scales (f).

\section{RESULTS AND DISCUSSION}

\section{(Co)Variance Components}

Genetic correlations used in runs 1 to 3 increased on average with increasing PDB (Table 3), because on average the estimated Ayrshire correlations were lower than the corresponding priors. Posterior means of genetic correlations inferred using BMACE were on average higher than the corresponding prior means and substantially higher than the genetic correlations used in runs 1 to 3 . The posterior means of genetic correlations were on average 0.02 units larger, when the PDB was low (i.e., 5 degrees of freedom) compared with high PDB (i.e., 100 degrees of freedom) for BMACE.

Posterior standard deviations of genetic correlations were on average less than 0.1 for 5 degrees of freedom and decreased with increasing PDB. Furthermore, posterior standard deviations of genetic correlations were higher for country combinations with few (e.g., Australia-United States) or no (e.g., Norway-United States) direct genetic links compared with country-pairs with several common bulls (e.g., Canada-United States). These 2 results were expected, because the posterior standard deviation is a function of both the PDB and information in data (formula [6]). The posterior distributions of genetic correlations were typically left skewed, but were closer to normal when the PDB increased (results not shown).

The differences between the genetic correlations obtained as a weighted average (formula [14]) and the corresponding posterior means from BMACE were 
Table 3. Three country-pair examples, mean, and range of across-country genetic correlations used in different multiple-trait across-country evaluation (MACE) runs (posterior standard deviation in subscript).

\begin{tabular}{|c|c|c|c|c|c|}
\hline Run & CAN-USA ${ }^{1}$ & AUS-USA ${ }^{2}$ & NOR-USA ${ }^{3}$ & Mean & Range \\
\hline 1 & 0.90 & 0.55 & 0.82 & 0.56 & 0.99 \\
\hline 2 & 0.91 & 0.64 & 0.79 & 0.60 & 0.85 \\
\hline 3 & 0.92 & 0.68 & 0.77 & 0.64 & 0.69 \\
\hline $10^{4}$ & $0.95_{0.02}$ & $0.82_{0.10}$ & $0.61_{0.16}$ & $0.79_{0.09}$ & $0.46_{0.16}$ \\
\hline $11^{4}$ & $0.95_{0.02}^{0.02}$ & $0.80_{0.08}$ & $0.63_{0.11}$ & $0.79_{0.07}$ & $0.43_{0.11}$ \\
\hline $12^{4}$ & $0.94_{0.01}$ & $0.78_{0.03}$ & $0.62_{0.05}$ & $0.77_{0.03}$ & $0.42_{0.05}$ \\
\hline Prior & 0.94 & 0.77 & 0.63 & 0.72 & 0.45 \\
\hline
\end{tabular}

${ }^{1} \mathrm{CAN}=$ Canada, USA $=$ United States; 61 and 495 common Ayrshire and Holstein bulls, respectively.

${ }^{2}$ AUS $=$ Australia, USA = United States; 6 and 197 common Ayrshire and Holstein bulls, respectively.

${ }^{3} \mathrm{NOR}=$ Norway, USA $=$ United States; zero common Ayrshire and Holstein bulls.

${ }^{4}$ Average posterior mean.

smaller for country-pairs with many direct genetic links compared with country-pairs having few or no direct genetic links. The differences in the mean of genetic correlations for all country-pairs were mainly due to differences in genetic correlations for country-pairs with few or no direct links (Mark et al., 2005).

The range of estimated genetic correlations between countries decreased with increasing PDB, and the range was less for posterior means from BMACE compared with the weighted averages used in run 1 to 3 . The reason for this was that many estimated genetic correlations were low between weakly linked countries, but when the PDB increased, the correlations increased and moved toward the average correlation.

Posterior means of heritabilities obtained from BMACE differed considerably from those used in national genetic evaluations and used in runs 1 to 6 (Table 4). However, it is not known what the correct values should be. Genetic parameters used in national genetic evaluations are often not frequently updated, and this may have some influence. Perhaps more importantly, parameters used in national evaluations do not necessarily correspond well to the data considered in international genetic evaluation, because pedigree information may differ, the data used in international genetic evaluations is a subset of the data used in national genetic evaluations and only approximate weighting factors are used in MACE. The heritabilities differed because of both estimated sire and residual variances being different for different methods.

Posterior standard deviations of heritabilities from BMACE ranged between 0.004 and 0.120 , tended to decrease with increasing population size, and decreased with increasing PDB. Posterior distributions of heritabilities and variances were approximately normal. Posterior means of residual covariances were all essentially zero, which was expected because only 1 trait was considered per country.

\section{Reliability and Prediction Error Variance}

The average reliability across all countries was $73 \%$ for domestic bulls regardless of the genetic correlations assumed in TMACE (runs 1 to 6 ). Reliabilities for breeding values on foreign scales increased with increasing genetic correlations; that is, the average reliability ranged from 34 in run 1 to $46 \%$ in run 4 for foreign bulls. Reliabilities for runs 7 to 9 were smaller than

Table 4. Comparison of assumed ${ }^{1}$ heritabilities for runs 1 to 6 and mean posterior ${ }^{2}$ heritabilities from runs 9 to 12 for the 9 countries $^{3}$ [average-information (AI)-REML ${ }^{4}$ estimates included for comparison].

\begin{tabular}{llllllllll}
\hline Run & AUS & CAN & DNK & FIN & GBR & NOR & NZL & SWE & USA \\
\hline 1 to 6 & 0.240 & 0.190 & 0.240 & 0.280 & 0.260 & 0.061 & 0.211 & 0.300 & 0.260 \\
9 and 12 & 0.242 & 0.196 & 0.267 & 0.180 & 0.235 & 0.040 & 0.200 & 0.242 & 0.264 \\
8 and 11 & 0.216 & 0.219 & 0.358 & 0.127 & 0.161 & 0.021 & 0.205 & 0.183 & 0.226 \\
7 and 10 & 0.202 & 0.237 & 0.438 & 0.120 & 0.130 & 0.017 & 0.221 & 0.179 & 0.209 \\
AI-REML & 0.243 & 0.211 & 0.361 & 0.097 & 0.079 & 0.010 & 0.211 & 0.126 & 0.129 \\
\hline
\end{tabular}

${ }^{1}$ The heritability provided by respective national genetic evaluation units were assumed and used to calculate residual variances in runs 1 to 6 .

${ }^{2}$ All genetic and residual (co)variance components are estimated (prior equal to assumed heritabilities in runs 1 to 6 ).

${ }^{3}$ Country codes: AUS $=$ Australia, CAN $=$ Canada, DNK $=$ Denmark, FIN $=$ Finland, GBR $=$ United Kingdom, NOR = Norway, NZL = New Zealand, SWE $=$ Sweden, USA $=$ United States.

${ }^{4}$ Algorithm of Madsen et al. (2000) was used. 
Table 5. Mean ${ }^{1}$ regression ${ }^{2}\left(b_{x \mid 1}\right)$ and correlation $\left(r_{x, 1}\right)$ coefficient between predicted genetic merit in run 1 and run $\mathrm{x}=\{2, \ldots, 12\}$ for domestic and foreign bulls, respectively.

\begin{tabular}{|c|c|c|c|c|}
\hline \multirow[b]{2}{*}{ Run } & \multicolumn{2}{|c|}{ Domestic } & \multicolumn{2}{|c|}{ Foreign } \\
\hline & $b_{\mathrm{x} \mid 1}$ & $\mathrm{r}_{\mathrm{x}, 1}$ & $b_{\mathrm{x} \mid 1}$ & $r_{x, 1}$ \\
\hline 2 & 1.00 & 1.00 & 1.03 & 0.99 \\
\hline 3 & 1.00 & 1.00 & 1.07 & 0.98 \\
\hline 4 & 1.00 & 1.00 & 1.20 & 0.94 \\
\hline 5 & 1.00 & 1.00 & 1.19 & 0.94 \\
\hline 6 & 1.00 & 1.00 & 1.17 & 0.95 \\
\hline 7 & 0.90 & 0.99 & 1.10 & 0.92 \\
\hline 8 & 0.91 & 0.99 & 1.10 & 0.93 \\
\hline 9 & 0.98 & 1.00 & 1.17 & 0.95 \\
\hline 10 & 0.90 & 0.98 & 1.32 & 0.62 \\
\hline 11 & 0.92 & 0.99 & 1.32 & 0.63 \\
\hline 12 & 0.97 & 1.00 & 1.35 & 0.66 \\
\hline
\end{tabular}

${ }^{1}$ Mean across all 9 countries.

${ }^{2}$ Regression coefficient for regression of predicted genetic merits from run $\mathrm{x}$ on predicted genetic merits from run 1 .

those for runs 4 to 6 for both foreign bulls and for domestic bulls, because of lower assumed heritabilities in runs 7 to 9 for the 2 largest populations.

Posterior prediction error variances from BMACE were substantially higher (often more than $1 \sigma_{\text {sire }}^{2}$ unit) compared with the approximate prediction error variances for TMACE (i.e., runs 7 to 9). The prediction error variances for TMACE were expected to be underestimated due to ignoring the uncertainty of dispersion parameters, and the underestimation was on average larger for foreign bulls compared with domestic bulls.

\section{International Genetic Merit}

Predicted international genetic merit for foreign bulls was generally more affected by different MACE methods and different genetic parameters compared with predicted international genetic merit for domestic bulls (Table 5). The impact of using different genetic correlations in TMACE (runs 1 to 6 ) was essentially zero for domestic bulls, whereas it caused larger differences for foreign bulls. The regression coefficient for the regression of predicted genetic merit from run $\mathrm{x}$ (where $\mathrm{x}=$ $2, . ., 6)$ on predicted genetic merit from run $1\left(\mathrm{~b}_{\mathrm{x} \mid 1}\right)$ and therefore also the standard deviation of predicted international genetic merit increased for foreign bulls with increasing genetic correlations. When the standard deviation of genetic merits increase for a certain group of bulls, these bulls are more likely to deviate from the population mean (e.g., rank high). The standard deviation of predicted international genetic merit increased with increasing PDB for both domestic and foreign bulls (runs 7 to 12). This can be explained by the increasing posterior means of heritabilities for Norway and Finland with increasing PDB (Table 4). Norway and Fin-
Table 6. Spearman rank (above diagonal) and Pearson productmoment (below diagonal) correlations between selected multiple-trait across-country evaluation (MACE) runs for top 100 ranking bulls. ${ }^{1}$ Mean correlation across all 9 countries is shown.

\begin{tabular}{lllll}
\hline & \multicolumn{5}{c}{ Run } \\
\cline { 2 - 5 } Run & 2 & 5 & 8 & 11 \\
\hline 2 & & 0.81 & 0.67 & 0.49 \\
5 & 0.90 & & 0.88 & 0.75 \\
8 & 0.85 & 0.93 & & 0.79 \\
11 & 0.64 & 0.81 & 0.82 & \\
\hline
\end{tabular}

${ }^{1}$ The top 100 bulls with data and a reliability value of at least $40 \%$ in run 1 were considered.

land had the largest Ayrshire populations and together they provided $54 \%$ of the total number of records included in the analyses (Table 1).

Comparisons of results from runs 10 to 12 with results from runs 7 to 9 clearly showed that accounting for the uncertainty of location parameters had a large impact on predicted international merit; that is, differences in mean genetic parameters alone could not explain differences in predicted genetic merit between BMACE and TMACE.

Trends in mean international predicted genetic merit per birth year of bulls differed considerably between TMACE and BMACE runs when all bulls, regardless of reliability, were considered. However, genetic trends were similar regardless of method when only bulls with a minimum reliability of $40 \%$ (run 1) were considered and trends did not depend on PDB. The large deviations in predicted international genetic merits between BMACE and run 1 were primarily for bulls with little information about their breeding values. The average product-moment correlation between predicted international genetic merits in run 1 and run 10 was 0.62 , $0.68,0.81$, and 0.88 when considering foreign bulls with a reliability in run 1 of at least $0,20,40$, and $60 \%$, respectively.

Australia and New Zealand generally had the largest changes in predicted genetic merit between different MACE approaches when all bulls regardless of reliability were considered. Both countries had relatively few bulls, weak genetic ties, and low genetic correlations with other countries; especially with the large Nordic Ayrshire populations. The majority of breeding values on the Australian scale had a reliability (in run 1) of less than $10 \%$ due to low correlations with Finland, Norway, and Sweden.

Bull rankings differed for TMACE and BMACE (Table 6) when considering the top 100 ranking bulls in run 1 with a reliability of at least $40 \%$. The mean Spearman rank correlation across all country scales ranged from 0.47 to 0.99 for all MACE runs. Pearson product-moment correlations were generally higher than Spear- 
Table 7. Mean square error (MSE) of difference between predicted national and international genetic merit from reduced multiple-trait across-country evaluation (MACE), regression coefficient from regressions of predicted genetic merit in full MACE on predicted genetic merit in reduced MACE $\left(\mathrm{b}_{\text {full lred }}\right)$, and correlation between predicted genetic merit in full MACE and predicted genetic merit in reduced MACE ( $\mathrm{r}_{\text {full,red }}$ ) for 3 different sets of bulls set to missing in 12 different reduced MACE runs both for the country scale where the national genetic merit was set to missing (d) and the 8 other country scales (f).

\begin{tabular}{|c|c|c|c|c|c|c|c|c|c|c|c|c|c|c|c|}
\hline \multirow[b]{2}{*}{ Run } & \multicolumn{5}{|c|}{$5 \%$ youngest } & \multicolumn{5}{|c|}{$5 \%$ random } & \multicolumn{5}{|c|}{$25 \%$ youngest imports } \\
\hline & MSE & $b_{\text {full|red }}^{d}$ & $r_{\text {full, red }}^{d}$ & $b_{\text {full|red }}^{f}$ & $\mathrm{r}_{\text {full, red }}^{\mathrm{f}}$ & MSE & $b_{\text {full|red }}^{d}$ & $\mathrm{r}_{\text {full, red }}^{\mathrm{d}}$ & $b_{\text {full|red }}^{f}$ & $\mathrm{r}_{\text {full, red }}^{\mathrm{f}}$ & MSE & $b_{\text {full|red }}^{d}$ & $\mathrm{r}_{\text {full, red }}^{\mathrm{d}}$ & $b_{\text {full|red }}^{f}$ & $\mathrm{r}_{\text {full, red }}^{\mathrm{f}}$ \\
\hline 1 to 2 & 0.55 & 1.00 & 0.69 & 1.03 & 0.86 & 0.66 & 0.95 & 0.67 & 0.97 & 0.81 & 0.43 & 1.12 & 0.87 & 1.01 & 0.97 \\
\hline 3 & 0.55 & 1.00 & 0.69 & 1.02 & 0.86 & 0.66 & 0.95 & 0.67 & 0.97 & 0.81 & 0.43 & 1.11 & 0.88 & 1.01 & 0.97 \\
\hline 4 & 0.55 & 1.00 & 0.69 & 1.02 & 0.84 & 0.66 & 0.95 & 0.67 & 0.97 & 0.80 & 0.48 & 1.07 & 0.90 & 1.02 & 0.97 \\
\hline 5 & 0.55 & 1.00 & 0.69 & 1.02 & 0.84 & 0.66 & 0.95 & 0.67 & 0.98 & 0.80 & 0.48 & 1.07 & 0.90 & 1.02 & 0.97 \\
\hline 6 & 0.55 & 1.00 & 0.69 & 1.02 & 0.84 & 0.66 & 0.95 & 0.67 & 0.97 & 0.80 & 0.48 & 1.08 & 0.89 & 1.02 & 0.97 \\
\hline 7 & 0.56 & 0.99 & 0.76 & 1.03 & 0.87 & 0.64 & 0.97 & 0.73 & 0.98 & 0.84 & 0.44 & 1.09 & 0.91 & 1.03 & 0.97 \\
\hline 8 & 0.56 & 0.99 & 0.76 & 1.03 & 0.87 & 0.65 & 0.97 & 0.73 & 0.98 & 0.84 & 0.45 & 1.09 & 0.91 & 1.03 & 0.97 \\
\hline 9 & 0.55 & 1.01 & 0.72 & 1.03 & 0.85 & 0.66 & 0.96 & 0.68 & 0.98 & 0.80 & 0.47 & 1.08 & 0.90 & 1.03 & 0.97 \\
\hline 10 & 0.54 & 1.01 & 0.77 & 1.00 & 0.97 & 0.63 & 0.99 & 0.75 & 0.97 & 0.97 & 0.57 & 0.93 & 0.87 & 0.95 & 0.96 \\
\hline 11 & 0.54 & 1.02 & 0.78 & 0.99 & 0.97 & 0.63 & 0.99 & 0.75 & 0.98 & 0.97 & 0.56 & 0.94 & 0.87 & 0.94 & 0.96 \\
\hline 12 & 0.53 & 1.03 & 0.75 & 1.01 & 0.97 & 0.63 & 0.98 & 0.73 & 0.98 & 0.97 & 0.58 & 0.93 & 0.86 & 0.93 & 0.96 \\
\hline
\end{tabular}

man rank correlations and ranged from 0.62 to 1.00 . The choice of PDB had a small impact on MACE predictions and bull rankings. Rank correlations ranged from 0.96 to 1.00 among runs 1 to 3 and from 0.98 to 1.00 among BMACE runs.

Treatment of genetic groups as either fixed or random effects could have an impact on predicted international genetic merit, but the group sizes were relatively large, so the impact is probably small.

Predictive ability. Differences in predictive ability between different MACE runs were, in most cases, small and the results did not clearly and consistently favor any of the investigated approaches although BMACE tended to perform best (Table 7). The largest difference in MSE between any 2 runs were 5.3, 5.4, and $30.3 \%$ for the subset of $5 \%$ youngest, $5 \%$ random, and $25 \%$ youngest imports, respectively. The relative differences were smaller for the regression and correlation coefficients. The mean absolute bias was always less than $0.65 \sigma_{\text {sire }}$ units, but single bulls had larger deviations between their national and predicted international genetic merit (up to $2.6 \sigma_{\text {sire }}$ units mean absolute bias). Regression of predicted genetic merit from full analysis on predicted genetic merit from reduced analysis indicated that all MACE models yielded predictions that were nearly unbiased, except for the youngest import bulls, where regression coefficients ranged from 0.93 to 1.12 .

Mean square error measures the consistency between international and national predictions. Low values of MSE indicate the best model, when national genetic merits are assumed unbiased. In practice, national genetic merits can be biased. Furthermore, international genetic merits on foreign country scales were most affected by differences in the MACE model. Thus, it is also useful to consider measures that do not compare directly with national predictions. Deviations of $b_{\text {full }}$ red from 1.0 indicates bias in international predictions ( Reverter et al., 1994). The correlation coefficient between predicted genetic merit in full and reduced MACE $\left(r_{\text {full,red }}\right)$ was also computed. This measure depends on both the precision and bias of evaluations. It measures the consistency of subsequent evaluations, which is an important practical property. Its expectation is less than 1.0, because the average reliability of predicted genetic merit is less for the reduced data compared with the full data (Reverter et al., 1994).

Young bulls typically only have daughter information in one country (i.e., the domestic country). The predicted genetic merit on the domestic scale for such a bull is due to daughter information in the domestic country and the within-country parent average. When the bull's national genetic merit is set to missing, its international genetic merit will become the withincountry parent average. Thus, the correlation matrix is not important in predicting the genetic merit of young bulls on their domestic country scale. This was the reason why comparisons on the domestic scale did not differ much for young bulls when genetic correlations differed (runs 1 to 6).

The genetic correlation matrix is crucial for the prediction of genetic merit of young bulls on foreign country scales. Besides information from parents, all information about the predicted genetic merit is typically correlated information from daughters in another country. This explains why the predictive ability of genetic merit on foreign country scales differed when different genetic correlations were used. The predictive ability of young bulls on foreign country scales tended to improve with lower average genetic correlation (runs 1 to 6).

The heritability determines the importance of pedigree information relative to information from progeny 
and is more important than genetic correlations for young bulls. This explains why using different heritabilities in TMACE (i.e., runs 7 to 9) had a larger impact on predictive ability than using different genetic correlations in TMACE (i.e., runs 1 to 6 ) and why both domestic and foreign scales were affected.

The MSE was nearly the same regardless of whether estimated or national heritabilities were assumed in TMACE. This was the case for both young and randomly chosen bulls. The $b_{\text {full, red }}$ indicated that using estimated instead of national heritabilities tended to increase bias for young bulls slightly. Contrarily, the bias for randomly chosen bulls tended to decrease for these bulls. Furthermore, the $r_{\text {full,red }}$ was clearly and consistently higher when estimated heritabilities were used in TMACE (runs 7 to 9) as opposed to national heritabilities (runs 4 to 6 ). This may be explained by the fact that estimated heritabilities decreased compared with heritabilities assumed in national evaluations for the 2 largest populations (Table 4). The weight on information from parents relative to information from daughters increase with decreasing heritabilities, so the impact of setting national evaluations to missing is smaller when heritabilities are low.

The MSE increased for young and randomly chosen bulls when the uncertainty of genetic parameters was taken into account (runs 10 to 12). Prediction bias for BMACE was slightly larger on the domestic scale for young bulls compared with TMACE. Prediction bias was, however, less for BMACE for randomly chosen bulls and on foreign country scales for both young and randomly chosen bulls. However, all MACE approaches were close to being unbiased for young bulls on both the domestic and foreign country scales (i.e., $b_{\text {full }}^{\mathrm{d}}$ red and $\mathrm{b}_{\text {full|red }}^{\mathrm{f}}$ ranged from 0.99 to 1.03). Predictions for randomly chosen bulls were also nearly unbiased (i.e., $b_{\text {full|red }}^{\mathrm{d}}$ ranged from 0.95 to 0.99 and $b_{\text {full|red }}^{f}$ ranged from 0.97 to 0.98 ).

The $r_{\text {full,red }}$ were substantially higher for BMACE compared with TMACE for both young and randomly chosen bulls. This was the case for both domestic and especially foreign country scales. Correlations were higher for foreign than domestic country scales. This is not because it is easier to predict genetic merit on foreign country scales, but because the relative difference in average reliability of predicted genetic merit between full and reduced analysis was larger for the domestic scale compared with the foreign scales.

Import bulls typically have national genetic merits in more than one country. The importance of local information relative to foreign information depends, besides genetic correlations, on the number of daughters and especially the number of local daughters (Mrode and
Swanson, 1999). Predictive information from daughters in other countries becomes relatively more important when the bull's national genetic merit is set to missing, and this can cause some substantial changes in the predicted international genetic merit on the domestic scale. The relatively high correlations $\left(\mathrm{r}_{\text {full,red }}^{\mathrm{f}}>0.95\right.$ and $r_{\text {full,red }}^{\mathrm{d}}>0.85$ ) between predicted genetic merit in full and reduced analysis showed that predicted genetic merit of import bulls was less affected by the information coming from the country where information was excluded.

The MSE for import bulls was lowest for TMACE and was lowest when genetic correlations were low. This result was not consistent with the results for young and randomly chosen bulls. The inconsistency could be due to the relatively few (27) chosen young import bulls. Another reason may be that national predictions for import bulls were biased because of preferential treatment of their daughters. Preferential treatment may occur when semen is expensive and progenies are considered more valuable than an average cow, which is typically the case for daughters of import bulls. High consistency of international and national genetic merit is not necessarily desirable if national predictions are biased. Such inconsistencies may instead indicate that the model used for international genetic evaluations was robust to biased national predictions. The slight superiority of BMACE in predicting unbiased international genetic merit for import bulls on the domestic country scale support this argument. Parent averages of import bulls on the domestic scale are less affected by setting national genetic merit of import bulls to missing when the genetic correlations are low. This may explain why national predictions were closer to international predictions (i.e., lower MSE) for import bulls when the across-country genetic correlations were low.

The difference between MSE and the variance of differences $\left(\sigma_{\text {nat-red }}^{2}\right)$ is equal to the squared average bias of the bulls in question. The MSE were 0.0 to $0.1 \%$ larger than $\sigma_{\text {nat-red }}^{2}$ for all runs when young and randomly chosen bulls were considered. Thus, all the MACE models considered had excellent extrapolation properties for these bulls. The MSE was 4 to $6 \%$ higher than $\sigma_{\text {nat-red }}^{2}$ for import bulls, which suggests that import bulls were slightly biased and that the extrapolation properties were not perfect for import bulls.

\section{General Discussion}

The main purpose of international genetic evaluations of dairy bulls is to accurately predict unbiased genetic merit of bulls in countries where the bull does not have (or has only limited) information from local 
daughters. Young bulls are usually the primary selection candidates, but the predicted international genetic merit of imported bulls in the country of importation is also of high interest. The BMACE approach seemed to perform best in all these situations. However, the superiority in predictive performance compared with TMACE was small and all MACE analyses yielded nearly unbiased predictions. Introducing any of the investigated MACE approaches for Ayrshire conformation traits would probably be better than having no international genetic evaluation. This is because Pearson product-moment and Spearman rank correlations were always positive, and genetic correlations differed from zero. Furthermore, in dairy cattle breeding, only breeding values associated with high reliabilities are typically published and the differences in predicted international genetic merit between methods decreased with increasing reliability. An important advantage of TMACE over BMACE is that it is computationally less demanding. A direct comparison of computational costs was not possible, but the traditional TMACE and REML approach was considerably faster despite being analyzed on a less powerful computer.

Priors. In retrospect, it would have been preferred to use sire variances from EM-REML estimation of all data rather than those from the well-connected subset as priors in BMACE, because the difference between sire variances estimated based on these 2 data sets was up to $51 \%$. However, the PDB for the prior genetic (co)variance matrix had only a relatively small impact on posterior means of heritabilities (Table 4).

Sigurdsson et al. (1996) argued in favor of keeping heritabilities fixed in estimating across-country correlations based on deregressed national breeding values. An important reason to assume national heritabilities is that their precision is higher than the precision of the heritabilities that can be estimated based on deregressed national genetic merits and their EDC. In a Bayesian context, keeping the heritabilities fixed is equivalent to having infinite high PDB for the ratio between sire and residual variances.

The expectation of heritabilities estimated from deregressed national genetic merits is not necessarily equal to the expectation of the heritabilities assumed in the national genetic evaluation. One reason for different heritabilities could be that the animals considered in the 2 evaluations are traced back to different base populations. The base population can differ due to differences in the pedigrees used in the 2 evaluations and due to EDC being poor approximations of the true reliabilities of national genetic evaluations. Pedigree information for domestic bulls is often more complete in national than international evaluations, whereas pedigree information on import bulls is often less complete at the national compared with the international level. Incomplete pedigree information on import bulls probably gives rise to a greater source of selection bias than incomplete pedigree information on bulls born in the domestic country. Another reason for different heritabilities could be that the multivariate estimation of heritabilities in BMACE reduces selection bias compared with estimation within country. In addition, the heritabilities used in national genetic evaluations are often not updated frequently.

The use of heritabilities different from the national ones in prediction of international genetic merit has important practical consequences. As a result, the deregression and MACE process is no longer reversible, and the predicted international genetic merits will not be expressed on the same scale as national genetic evaluations. This makes calculation of total merit indexes and blending of international genetic evaluation results with national genetic evaluation results for cows and bulls not included in the international evaluation problematic.

In principle, the EDC should also be rescaled according to the re-estimated heritabilities during iteration when heritabilities different from those assumed in national genetic evaluations are used in MACE. This could be done after deregression while sire variances are being estimated. However, more information than was available for this study would be needed to separate contributions from daughters and their maternal ancestors.

It could be argued that a fair comparison of bulls across countries is more important than a fair comparison of bulls and cows within country. However, it was observed here that the predictive performance of MACE is quite robust to different genetic parameters (e.g., small differences in predictive ability for runs 1 to 9). Although estimated and national heritabilities differed considerably, the overall predictive ability of TMACE was only slightly better when estimated heritabilities were considered instead of the national ones. Therefore, for practical purposes, we recommend the use of national heritabilities, whereas estimated heritabilities may be used for validation purposes.

Predictive ability measures. In principle, daughter information should have been excluded in national genetic evaluations for bulls having their national genetic merit excluded in MACE for the purpose of predictive ability assessment. However, this was not possible, as it would have required the involved countries to rerun their national evaluation. Furthermore, excluding daughter information for these bulls in the national evaluation would be expected to have a limited effect on the predicted genetic merit of other bulls and therefore also on the predictive ability measures. 
It would be possible to circumvent the problem of national predictions containing information due to the bulls set to missing by applying the deregression to the full data set. Hence, all information due to the bulls set to missing would be removed from the national predictions. The predicted international genetic merits of the selected bulls would then be entirely due to correlated information from other countries and remote ancestors considered in the national evaluation, but not in MACE. We preferred not to remove the influence of the within-country parent average in the country in question, because the predicted international genetic merit of most young bulls would otherwise essentially be based on no information.

In the data set with the national genetic merit of some import bulls set to missing, 33 pairwise links were lost, of which 2 links were due to the same common bull having evaluations in more than 2 countries. The BMACE could therefore be expected to perform more poorly for this data set, because genetic parameters were resampled. Parameters based on the data sets with excluded information should have been used for TMACE to enable a fair comparison between BMACE and TMACE for the import bulls. However, this was not done, because the estimation of genetic correlations with EM-REML was problematic when many of the genetic ties were excluded (i.e., the average genetic correlation was 0.12 for the data set with $25 \%$ imports set to missing). In comparison, differences in posterior mean genetic correlations between the full and reduced BMACE runs were always very small and typically smaller than the posterior standard deviations (due to strong dependency on prior genetic correlations).

The choice of number of national genetic evaluations to leave out for determining the predictive ability of the model was somewhat arbitrary. It was a compromise between having sufficient information to both estimate model parameters and to accurately measure the predictive ability. Shao (1993) showed that predictive ability measure (e.g., MSE) is asymptotically inconsistent when the number of observations left out is small. The full data set is often split in 2 balanced sets with similar statistical properties to ensure good extrapolation properties (e.g., McCarthy, 1976; Snee, 1977). In this study, the lack of connectedness was critical in estimating all model parameters, so we only left out $5 \%$ or less of national genetic merits. Furthermore, we were interested in the predictive ability of specific target animals and extrapolation properties were generally quite good.

Further improvements of BMACE. The BMACE may be further improved by considering genetic groups as random effects to allow for smaller group sizes. Variances might also be heterogeneous within countries; for example, for different birth year classes. This was ignored here to limit the scope of the study, but options to accommodate heterogeneous variances have been implemented in the software used. The model may also be improved by specifying heterogeneous PDB and by keeping certain parameters (e.g., heritabilities and residual correlations) fixed.

Schaeffer (2001) suggested an approximate Bayesian estimation of (co)variances via Gibbs sampling, where posterior residual variances are replaced by the prior residual variances after each round of iteration. Elaborating on this idea, heterogeneous realized PDB could be assigned to prior (co)variance matrices by replacing the "posterior" samples with a weighted average of prior and the sampled "posterior" from the inverse-Wishart distribution. This would allow PDB for variances not to be infinitively high while still being higher than the PDB for correlations or vice versa. Such an approach might be desirable when prior (co)variances are associated with different PDB. This was the case here, although ignored in our Bayesian implementation. A more elegant solution would be to assign prior (co)variances with heterogeneous PDB. Then other prior distributions such as normal or $\mathrm{t}$ distributions need to be specified. This would, however, involve sampling by $\mathrm{Me}-$ tropolis-Hastings algorithm due to complicated distributional properties for posterior (co)variances.

It may be of higher practical interest to investigate solutions to reduce the computational costs in the short term. The current implementation of the Gibbs sampler used in BMACE uses a relatively simple blocking strategy, where each block consists of all equations belonging to a single bull. A much more efficient strategy might be to sample all location parameters simultaneously (García-Cortés and Sorensen, 1996). This will require solving the mixed model equations in each round of the Gibbs sampler. The Langevin-Hastings algorithm (Besag, 1994) is one option, which could be explored to sample location and dispersion parameters simultaneously. Computation time for BMACE may also be reduced by distributing the computations on a number of different processors (Larsen and Madsen, 1999).

Implications. We have shown that better predictive performance can be gained by considering prior information and uncertainty of (co)variances in MACE for weakly linked populations. This result is of special interest to populations that are in their early stages of cooperation whereas less can be gained for populations that already have many genetic ties.

The TMACE assuming a simple weighted average of prior and estimated Ayrshire genetic correlations $(d=$ 4) was implemented for routine evaluations of Ayrshire conformation traits in November 2003 based on results of this study. The reason for this was that the superior- 
ity in predictive performance of BMACE compared with TMACE was small for bulls with moderate to high reliability, whereas BMACE was much more computer demanding than TMACE. The intermediate PDB was chosen, because it seemed to yield more meaningful genetic correlations than pure Ayrshire estimates, thus enabling greater international cooperation among populations. A similar approach has subsequently been applied to international genetic evaluations for other breeds and traits. Trait definitions for other traits such as milk production and SCC are usually better harmonized across breeds within country. This would allow higher PDB to be specified compared with conformation traits.

\section{CONCLUSIONS}

A fully Bayesian MACE was applied to 9 weakly connected bull populations. The overall predictive ability of this model was slightly superior to traditional MACE assuming a weighted average of prior and estimated genetic correlations. The overall predictive ability of a fully Bayesian MACE was also slightly superior to traditional MACE assuming posterior means of genetic parameters inferred with Bayesian MACE. The choice of PDB had a small impact on predicted international genetic merit on foreign country scales. Sire rankings differed depending on method of analysis and especially for bulls with low reliability (e.g., on foreign country scales). All MACE analyses yielded nearly unbiased predictions and could be used for international genetic evaluations of Ayrshire conformation. Reliabilities for traditional MACE were overestimated due to the assumption of known parameters.

\section{ACKNOWLEDGMENTS}

We acknowledge the 9 national genetic evaluation units for providing data, Bert Klei for earlier discussion about weighting of separate correlation estimates, and the Danish Centre for Scientific Computing for supplying the computer capacity used for BMACE.

\section{REFERENCES}

Besag, J. 1994. Contribution to the discussion paper by Grenander and Miller. J. Royal Stat. Soc. B 56:591-592.

Fikse, W. F., and G. Banos. 2001. Weighting factors of sire daughter information in international genetic evaluations. J. Dairy Sci. 84:1759-1767.

García-Cortés, L. A., and D. Sorensen. 1996. On a multivariate implementation of the Gibbs sampler. Genet. Sel. Evol. 28:121-126.

Gianola, D., and R. L. Fernando. 1986. Bayesian methods in animal breeding theory. J. Anim. Sci. 63:217-244.

Harris, B., and D. Johnson. 1998. Information source reliability method applied to MACE. Proc. 1998 Interbull Mtg., Rotorua, New Zealand. Interbull Bull. 17:31-36.
Interbull. 2002. Interbull routine genetic evaluation for conformation traits, February 2002. Online. Available www.interbull.org/ GeneticEvaluations/conformation/February2002. Accessed Apr. $2,2005$.

Jairath, L., J. C. M. Dekkers, L. R. Schaeffer, Z. Liu, E. B. Burnside, and B. Kolstad. 1998. Genetic evaluation for herd life in Canada. J. Dairy Sci. 81:550-562.

Jensen, J., and P. Madsen. 2002. Bayesian estimation of within and across country genetic parameters for MACE. Proc. 2002 Interbull Mtg., Interlaken, Switzerland. Interbull Bull. 29:18-21.

Jensen, J., C. S. Wang, D. A. Sorensen, and D. Gianola. 1994. Bayesian inference on variance and covariance components for traits influenced by maternal and direct genetic effects, using the Gibbs sampler. Acta Agric. Scand. A Anim. Sci. 44:193-201.

Jorjani, H., L. Klei, and U. Emanuelson. 2004. A simple method for weighted bending of genetic (co)variance matrices. J. Dairy Sci. 86:677-679.

Klei, B., and T. Lawlor. 2001. Mace for conformation, Ayrshires. Proc. 2001 Interbull Mtg., Budapest, Hungary. Interbull Bull. 27:51-55.

Klei, B., and K. A. Weigel. 1998. A method to estimate correlations among traits in different countries using data on all bulls. Proc. 1998 Interbull Mtg., Rotorua, New Zealand. Interbull Bull. 17:8-14.

Larsen, M., and P. Madsen. 1999. The CEBUS project: History and overview. Interbull Bull. 20:3-10.

Madsen, P., and J. Jensen. 2003. A user's guide to DMU. A package for analysing multivariate mixed models. Version 6 , release 4 . Danish Institute of Agricultural Sciences, Foulum, Denmark.

Madsen, P., J. Jensen, and T. Mark. 2000. Reduced rank estimation of (co)variance components for international evaluation using AIREML. Proc. 2000 Interbull Mtg., Bled, Slovenia. Interbull Bull. 25:46.

Mark, T., P. Madsen, J. Jensen, and W. F. Fikse. 2005. Short communication: Difficulties in estimating across country genetic correlations for weakly linked bull populations. J. Dairy Sci. 88:33033305 .

McCarthy, P. J. 1976. The use of balanced half-sample replication in cross-validation studies. J. Am. Stat. Assoc. 71:596-604.

Mrode, R. A., and G. J. T. Swanson. 1999. Simplified equations for evaluations of bulls in the Interbull international evaluation system. Livest. Prod. Sci. 61:43-52.

Reverter, A., B. L. Golden, R. M. Bourdon, and J. S. Brinks. 1994. Technical note: Detection of bias in genetic predictions. J. Anim. Sci. 72:34-37.

Shao, J. 1993. Linear model selection by cross-validation. J. Am. Stat. Assoc. 88:486-494.

Snee, R. D. 1977. Validation of regression models: Methods and examples. Technometrics 19:415-428.

Schaeffer, L. R. 1994. Multiple-country comparisons of dairy sires. J. Dairy Sci. 77:2671-2678.

Schaeffer, L. R. 2001. Multiple trait international bull comparisons. Livest. Prod. Sci. 69:145-153.

Schenkel, F. S., L. R. Schaeffer, and P. J. Boettcher. 2002. Comparison between estimation of breeding values and fixed effects using Bayesian and empirical BLUP estimation under selection on parents and missing information. Genet. Sel. Evol. 34:41-59.

Scott, D. W. 1992. Multivariate density estimation: Theory, practice, and visualization. Wiley Series in Probability and Mathematical Statistics. John Wiley, New York, NY.

Sigurdsson, A., G. Banos, and J. Philipsson. 1996. Estimation of genetic (co)variance components for international evaluation of dairy bulls. Acta. Agric. Scand. A Anim. Sci. 46:129-136.

Sullivan, P. G. 1999. Appendix: REML estimation of heterogeneous sire (co)variances for MACE. Interbull Bull. 22:146-148.

Van Tassell, C. P., and L. D. Van Vleck. 1996. Multiple-trait Gibbs sampler for animal models: Flexible programs for Bayesian and likelihood-based (co)variance component inference. J. Anim. Sci. 74:2586-2597. 